\title{
Dually Normal ADL
}

\author{
S. Ravi Kumar ${ }^{1}$ \\ Reader, Dept. of Mathematics \\ M.R.College(A), \\ Vizianagaram, India
}

\author{
G.C.Rao ${ }^{2}$ \\ Department of Mathematics \\ Andhra University \\ Visakhapatnam, India
}

\begin{abstract}
The concept of Normal ADL was introduced in my earlier paper Normal Almost Distributive Lattices[2]. In this Paper, we introduce the concept of a dually normal ADL and we characterize Dually Normal ADLs in terms of dual annihilators. Throughout this Chapter we consider ADL $R$ means an ADL with at least one maximal element.
\end{abstract}

Keywords: Almost Distributive Lattice(ADL), Normal ADL, Dual annihilators, Dually Normal ADL.

\section{Introduction}

The concept of Normal lattice was introduced by W.H.Cornish [4] as a distributive lattice with 0 in which every prime ideal contains a unique minimal prime ideal. In [2], we introduced the concept of an ADL $R$ being normal through its principal ideal lattice $P I(R)$. That is, an ADL $R$ with 0 is a Normal Almost Distributive Lattice if its principal ideal lattice $P I(R)$ is a normal lattice.

\section{DUal ANNiHILATors}

In this section, we introduce a dual annihilator and study some of its properties. First we start with the following definition.

1.1. Definition : Let $R$ be an ADL with maximal elements and $S$ be any non-empty subset of $R$. Define $(S)^{+}=\{x \in R \mid s \vee x$ is maximal for all $s \in S\}$.

On routine verification, we can prove the following result.

1.2. Lemma : For any $a, b \in R$, if $a \vee b$ is a maximal element in $R$ then for any $x \in R$, the elements $x \vee a \vee b$ and $a \vee x \vee b$ are also maximal in $R$.

In the following theorem, we prove that the set $(S)^{+}$is a filter in $R$.

1.3. Theorem : For any non-empty subset $S$ of $R$, the set $(S)^{+}$is a filter of $R$

Proof : Let $S$ be any non-empty subset of an ADL $R$ and $a, b \in(S)^{+}$.

Then for any $s \in S$, the elements $s \vee a, s \vee b$ are maximal in $R$.

Consider the element $s \vee(a \wedge b)$, for some $s \in S$.

Now, for any $x \in R,[s \vee(a \wedge b)] \wedge x=(s \vee a) \wedge(s \vee b) \wedge x=(s \vee a) \wedge x)=x$.

Therefore $a \wedge b \in(S)^{+}$. Again, let $x \in R$ and $s \in(S)^{+}$. Clearly, $s \vee a$ is maximal for every $s \in S$, and hence the element $s \vee(x \vee a)$ is maximal for every $x \in R$. Therefore, $x \vee a \in(S)^{+}$. Therefore $(S)^{+}$is a filter in $R$.

In the following, we define a dual annihilator in an ADL $R$. 
1.4. Definition : If $S=\{s\}$ in the above theorem, then we write $(S)^{+}=(s)^{+}$instead of $(\{s\})^{+}$and we have $(s)^{+}=\{x \in R \mid s \vee x$ is maximal in $R\}$. Clearly, $(s)^{+}$is a filter in $R$ and we call this filter as a dual annihilator of $s$ in $R$.

In the following theorem, we prove some important properties of dual annihilators in an ADL $R$

1.5. Theorem : Let $R$ be an ADL with maximal element. Then for any $a, b$ in $R$
1). $a \leq b \Rightarrow(a)^{+} \subseteq(b)^{+}$
2). $(a \vee b)^{+}=(b \vee a)^{+}$
3). $(a \wedge b)^{+}=(b \wedge a)^{+}$
4). $(a \wedge b)^{+}=(a)^{+} \cap(b)^{+}$
5). $(a)^{+} \vee(b)^{+} \subseteq(a \vee b)^{+}$
6). $a \in(x)^{+} \Rightarrow(x)^{++} \subseteq(a)^{+}$
7). $a \in[b) \Rightarrow(b)^{+} \subseteq(a)^{+}$
8). $(a] \subseteq(b] \Rightarrow(b)^{+} \subseteq(a)^{+}$

\section{Proof :}

(1) : Let $a, b \in R$ such that $a \leq b$. Then $a \vee b=b=b \vee a$.

Now, $x \in(a)^{+} \Rightarrow a \vee x$ is maximal in $R \Rightarrow b \vee a \vee x$ is maximal in $R$

$$
\begin{aligned}
& \Rightarrow b \vee x \text { is maximal (since } b \vee a=b \text { ) } \\
& \Rightarrow x \in(b)^{+}
\end{aligned}
$$

Therefore $(a)^{+} \subseteq(b)^{+}$

(2) : Let $a, b$ be any two elements of $R$. Now, $x \in(a \vee b)^{+} \Leftrightarrow a \vee b \vee x$ is maximal in $R$ $\Leftrightarrow b \vee a \vee x$ is maximal in $R \quad \Leftrightarrow x \in(b \vee a)^{+}$. Therefore $(a \vee b)^{+}=(b \vee a)^{+}$

(3) : Let $a, b$ be any two elements of $R$. Now, $x \in(a \wedge b)^{+} \Leftrightarrow(a \wedge b) \vee x$ is maximal in $R$ $\Leftrightarrow(b \wedge a) \vee x$ is maximal in $R \Leftrightarrow x \in(b \wedge a)^{+}$. Therefore $(a \wedge b)^{+}=(b \wedge a)^{+}$

(4) : Let $a, b$ be any two elements of $R$.

We have $a \wedge b \leq b \Rightarrow(a \wedge b)^{+} \subseteq(b)^{+} \quad($ from (1))

Similarly, $b \wedge a \leq a \Rightarrow(b \wedge a)^{+} \subseteq(a)^{+} \Rightarrow(a \wedge b)^{+} \subseteq(a)^{+} \quad$ (from (3)).

Therefore $(a \wedge b)^{+} \subseteq(a)^{+} \cap(b)^{+}$.

$$
\text { Again } \begin{aligned}
x \in(a)^{+} \cap(b)^{+} & \Rightarrow x \in(a)^{+} \text {and } x \in(b)^{+} \\
& \Rightarrow a \vee x \text { and } b \vee x \text { are maximal in } R \\
& \Rightarrow x \vee a \text { and } x \vee b \text { are maximal in } R \\
& \Rightarrow(x \vee a) \wedge(x \vee b) \text { is maximal in } R \\
& \Rightarrow x \vee(a \wedge b) \text { is maximal in } R \Rightarrow(a \wedge b) \vee x \text { is maximal in } R \\
& \Rightarrow x \in(a \wedge b)^{+} \text {Therefore }(a)^{+} \cap(b)^{+} \subseteq(a \wedge b)^{+} .
\end{aligned}
$$

Hence $(a \wedge b)^{+}=(a)^{+} \cap(b)^{+}$ 
(5) : Proof follows from (1) and (2).

(6) : Let $a, x$ be any two elements of $R$. Suppose $a \in(x)^{+}$

Now $t \in(x)^{++} \Rightarrow s \vee t$ is maximal for all $s \in(x)^{+}$.

$\Rightarrow a \vee t$ is maximal in $R\left(\right.$ since $\left.a \in(x)^{+}\right) \Rightarrow t \in(a)^{+}$.

Therefore $(x)^{++} \subseteq(a)^{+}$

(7) : Let $a, b$ be any two elements of $R$. Suppose $a \in[b)$. Then $a \vee b=a$.

Now $t \in(b)^{+} \Rightarrow b \vee t$ is maximal in $R$

$\Rightarrow a \vee b \vee t$ is maximal

$\Rightarrow a \vee t$ is maximal in $R($ since $a \vee b=a) \Rightarrow t \in(a)^{+}$.

Therefore $(b)^{+} \subseteq(a)^{+}$.

(8) : Proof follows from (7).

\section{DUALly Normal ADLs}

In this section we introduce a dually normal and we characterize dually normal ADLs in terms of dual annihilators and in terms of maximal elements.

First we define a dually normal ADL in the following.

2.1. Definition : An ADL $R$ with maximal element is said to be dually normal if for any prime filter $F$ of $R, C(F)=\{x \in R \mid y \vee x$ is maximal, for some $y \notin F$ is a prime filter of $R$.

In the following theorem, we characterize a dually normal ADL in terms of dual annihilators. II

2.2. Theorem : Let $R$ be an ADL with maximal elements. Then the following are equivalent.

1). $R$ is dually normal.

2). For any $x, y \in R$, if $x \vee y$ is maximal then $(x)^{+} \vee(y)^{+}=R$

3). For any $x, y \in R,(x \vee y)^{+}=(x)^{+} \vee(y)^{+}$

\section{Proof :}

(1) $\Rightarrow$ (2) : Assume that $R$ is a dually normal ADL. Let $x, y \in R$ such that $x \vee y$ is maximal. We have to prove that $(x)^{+} \vee(y)^{+}=R$. Suppose $(x)^{+} \vee(y)^{+} \neq R$. Then there exists a maximal filter $G$ of $R$ such that $(x)^{+} \vee(y)^{+} \subseteq G$. Since $G$ is a prime filter and $R$ is dually normal, we have $C(G)$ is a prime filter of $R$. Therefore $x \vee y \in C(G)$ implies that either $x \in C(G)$ or $y \in C(G)$. If $x \in C(G)$ , then by definition, there exists some $t \notin G$ such that $x \vee t$ is maximal and hence $t \in(x)^{+} \subseteq G$. This is a contradiction. Therefore $x \notin G$. Similarly, we get $y \notin G$. Thus we get $x \vee y \notin G$. This is a contradiction. Therefore we get $(x)^{+} \vee(y)^{+}=R$.

(2) $\Rightarrow(3):$ Let $x, y \in R$. We have to prove that $(x)^{+} \vee(y)^{+}=(x \vee y)^{+}$

From (5) of Lemma 1.2, we have $(x)^{+} \vee(y)^{+} \subseteq(x \vee y)^{+}$.

Now, $\quad t \in(x \vee y)^{+} \Rightarrow x \vee y \vee t$ is maximal

$\Rightarrow t \vee x \vee y$ is maximal

$\Rightarrow t \vee x \vee t \vee y \quad$ is maximal

$\Rightarrow(t \vee x)^{+} \vee(t \vee y)^{+}=R$ from condition (2). 
Now $t \in R \Rightarrow t=s_{1} \wedge s_{2}$ where $s_{1} \in(t \vee x)^{+}$and $s_{2} \in(t \vee y)^{+}$.

$$
\begin{aligned}
& \Rightarrow t=s_{1} \wedge s_{2} \text { where } t \vee x \vee s_{1} \text { and } t \vee y \vee s_{2} \text { are maximal in } R . \\
& \Rightarrow t=s_{1} \wedge s_{2} \text { where } x \vee t \vee s_{1} \text { and } y \vee t \vee s_{2} \quad \text { are maximal in } R . \\
& \Rightarrow t=s_{1} \wedge s_{2} \text { where } t \vee s_{1} \in(x)^{+} \text {and } t \vee s_{2} \in(y)^{+}
\end{aligned}
$$

Therefore $t=t \vee\left(s_{1} \wedge s_{2}\right)=\left(t \vee s_{1}\right) \wedge\left(t \vee s_{2}\right) \in(x)^{+} \vee(y)^{+}$

Thus $(x \vee y)^{+} \subseteq(x)^{+} \vee(y)^{+}$and hence $(x \vee y)^{+}=(x)^{+} \vee(y)^{+}$

(3) $\Rightarrow$ (1) : Assume condition (3). Let $F$ be a prime filter of $R$. We have to prove that $R$ is dually normal. From Lemma 2.1.5, we have $C(F)$ is a filter of $R$.

Now, let $x, y \in R$ and $x \vee y \in C(F)$. Then $t \vee x \vee y$ is maximal for some $t \notin F$.

Now $t \vee x \vee y$ is maximal $\quad \Rightarrow x \vee y \vee t$ is maximal

$$
\begin{aligned}
& \Rightarrow t \in(x \vee y)^{+} \\
& \Rightarrow t \in(x)^{+} \vee(y)^{+} \quad[\text { from (3)] } \\
& \Rightarrow t=t_{1} \wedge t_{2} \quad \text { where } t_{1} \in(x)^{+}, t_{2} \in(y)^{+} \\
& \Rightarrow t=t_{1} \wedge t_{2} \quad \text { where } x \vee t_{1}, \quad y \vee t_{2} \text { are maximal in } R . \\
& \Rightarrow t=t_{1} \wedge t_{2} \quad \text { where } t_{1} \vee x, t_{2} \vee y \text { are maximal in } R .
\end{aligned}
$$

Since $F$ is prime and $t=t_{1} \wedge t_{2} \notin F$, we get $t_{1} \notin F$ or $t_{2} \notin F$.

Suppose $t_{1} \notin F$. Since $t_{1} \vee x$ is maximal and $t_{1} \notin F$, we get $x \in C(F)$.

Similarly, if $t_{2} \notin F$, we get $y \in C(F)$. Thus, we have $C(F)$ is a prime filter of $R$.

We conclude this chapter with the following theorem in which we characterize a dually normal $\mathrm{ADL}$ in terms of maximal elements.

2.3. Theorem : An ADL $R$ is dually normal if and only if for $x, y \in R$, if $x \vee y$ is maximal then there exist $x_{1}, y_{1} \in R$ such that $x \vee x_{1}, y \vee y_{1}$ are maximal in $R$ and $x_{1} \wedge y_{1}=0$.

Proof : Assume that $R$ is dually normal. Let $x, y \in R$ and $x \vee y$ is maximal in $R$. Then from Theorem 2.2, we have $(x)^{+} \vee(y)^{+}=R$.

Now, $0 \in R \Rightarrow 0=x_{1} \wedge y_{1} \quad$ where $x_{1} \in(x)^{+}$and $y_{1} \in(y)^{+} \quad \Rightarrow 0=x_{1} \wedge y_{1} \quad$ and $x \vee x_{1}$, $y \vee y_{1}$ are maximal in $R$. That is, if for any $x, y \in R$ such that $x \vee y$ is maximal, then there exist $x_{1}, y_{1} \in R$ such that $x \vee x_{1}, y \vee y_{1}$ are maximal in $R$ and $x_{1} \wedge y_{1}=0$.

Conversely, assume the given condition.

Let $x, y \in R$ and $x \vee y$ is maximal in $R$. Now we prove that $(x)^{+} \vee(y)^{+}=R$.

Since $x \vee y$ is maximal, from our assumption, there exist $x_{1}, y_{1} \in R$ such that $x \vee x_{1}, y \vee y_{1}$ are maximal in $R$ and $x_{1} \wedge y_{1}=0$.

Clearly, $x_{1} \in(x)^{+}$and $y_{1} \in(y)^{+}$and hence $0=x_{1} \wedge y_{1} \in(x)^{+} \vee(y)^{+}$

Now, $\quad 0 \in(x)^{+} \vee(y)^{+} \Rightarrow[0) \subseteq(x)^{+} \vee(y)^{+} \Rightarrow R \subseteq(x)^{+} \vee(y)^{+}$.

Therefore $R=(x)^{+} \vee(y)^{+}$. Thus $R$ is dually normal. 


\section{REFERENCES}

[1] Rao, G.C. and Ravi Kumar, S., Minimal prime ideals in Almost Distributive Lattices, International Journal of Contemporary Mathematical Sciences, Vol. 4, 2009, no. 10, 475 - 484.

[2] Rao, G.C. and Ravi Kumar, S. , Normal Almost Distributive Lattices, Southeast Asian Bulletin of Mathematics. 32(2008), 831 - 841.

[3] Swamy, U.M. and Rao,G.C. , Almost Distributive Lattices, Journal of Australian Mathematical Society., (Series A),31 (1981),77-91.

[4] William H. Cornish, Normal Lattices ,J.Austral. Math. Soc. 16(1972), 200-215.

[5] Ravi Kumar, S., Normal Almost Distributive Lattices, Doctoral Thesis (2010), Dept.of Mathematics, M.R.College(A), Vizianagaram.

[6] Swamy, U.M. and Rao,G.C., Almost Distributive Lattices, Journal of Australian Mathematical Society., (Series A),31 (1981),77-91.

[7] William H. Cornish, Normal Lattices, J.Austral. Math. Soc. 16(1972), 200-215.

\section{AUTHORS' BIOGRAPHY}

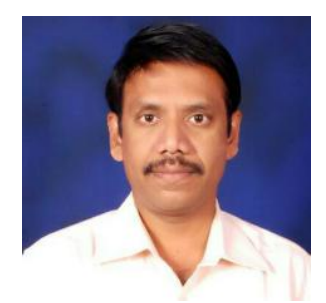

Lt. Dr. S. Ravi Kumar: He is presently working as Head \& Associate Professor in the Department of Mathematics, Maha Rajah's Autonomous College, Vizianagaram. He obtained his Ph.D Degree on "Normal Almost Distributive Lattices" in Lattice Theory. His yeoman services as a lecturer, Head of the Mathematics Department, Controller of Examinations, Students Training \& Placement Officer and as an Associate NCC Officer, made him a role model to thousands of students . He has one M.Phil in his credit. His papers more than 6 were published in various esteemed reputable Journals. He is a Member of Various Professional Bodies.

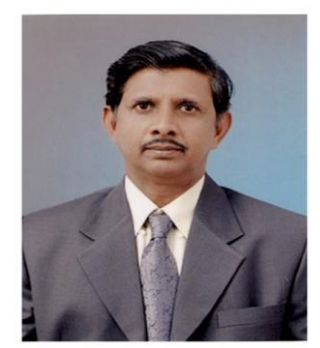

G.Chakradhara Rao: $\mathrm{He}$ is working as a Professor in the Department of Mathematics, Andhra University (AU), Visakhapatnam which is a famous and reputed university of Andhra Pradesh. He obtained his Ph.D Degree in Mathematics from AU under the able guidance of Prof.U.M.Swamy. He has participated in many seminars and presented his papers. He has nearly $12 \mathrm{Ph} . \mathrm{Ds}$ and 13 M.phils to his credit. More than 66 articles were published in various National and International Journals. He authored 8 books on various topics in Mathematics. 